\title{
Link Budgets: How Much Energy is Really Received
}

\author{
Aarne Mämmelä ${ }^{1}$, Adrian Kotelba1, \\ Marko Höyhtyä ${ }^{1}$ and Desmond P. Taylor ${ }^{2}$ \\ ${ }^{1}$ VTT Technical Research Centre of Finland \\ ${ }^{2}$ University of Canterbury \\ ${ }^{1}$ Finland \\ ${ }^{2}$ New Zealand
}

\section{Introduction}

In this chapter we consider various factors affecting link power or energy budgets, which are introduced in some detail for mobile and satellite communications in (Saunders, 1999, pp. 97-99; Sklar, 2001, pp. 242-303). We use the link budget as presented in (Saunders, 1999) for mobile cellular systems as a starting point. Some loss factors may be random. If we do not know the distributions of those random variables, it is reasonable to use the worst case analysis (French, 1986, p. 36), which is useful but in general highly pessimistic. We discuss the alternatives based on decision theory. In some cases it is reasonable to compute link budgets for the average performance instead of worst case performance. We can also use outage probabilities in the worst case analysis.

The goal of a link budget is to estimate the received signal-to-noise ratio (SNR) per bit at the input of the combiner/equalizer in the receiver (Saunders, 1999; Sklar, 2001), as in Fig. 1. Normally the combiner/equalizer includes some kind of correlator, which tries to use the received signal in the best possible way (Proakis, 2001).

It has been known at least since the mid-1960's that the transmitter power or energy is a basic system resource (Lucky et al., 1968, pp. 51-58; Schwartz et al., 1966, pp. 555-558). The different loss factors are assumed to be multiplicative in the linear domain and additive in the logarithmic domain, i.e., in decibels. In addition, the additive noise level is estimated by measuring the receiver noise floor and certain link margins are added. Most of the additive noise actually arises in the receiver front end. If there are several antennas, the sum of the powers is used. With a comprehensive link budget the necessary design trade-offs can be made in a systematic way before the system is actually built.

Link budgets do not include phenomena in or preceding the power amplifier, for example its finite efficiency. We do not discuss transmitter and receiver power budgets, i.e., how much energy or power is actually taken from the battery or electricity network. There are also other link budgets such as delay budgets that we do not consider.

Many loss factors depend on the frequency range (Saunders, 1999; Chu \& Greenstein, 1999). The wavelength is $\lambda=c / f$ where $c$ is the propagation velocity of the radio waves and $f$ is the frequency. Radio frequencies range from $3 \mathrm{kHz}$ to $300 \mathrm{GHz}$ with wavelengths from $100 \mathrm{~km}$ down to $1 \mathrm{~mm}$. The use of frequencies below $3 \mathrm{kHz}$ is in general impractical and above 300 
$\mathrm{GHz}$ we have infrared frequencies. In many modern mobile communication systems the frequencies employed are between $1 \mathrm{GHz}$ and $10 \mathrm{GHz}$, and the wavelengths are then between $30 \mathrm{~cm}$ and $3 \mathrm{~cm}$. We will assume that the antennas are used only in the far field, which implies that the dimensions of all antennas are small compared to the distance between transmitter and receiver antennas. Thus we will not consider any evanescent or near field effects.

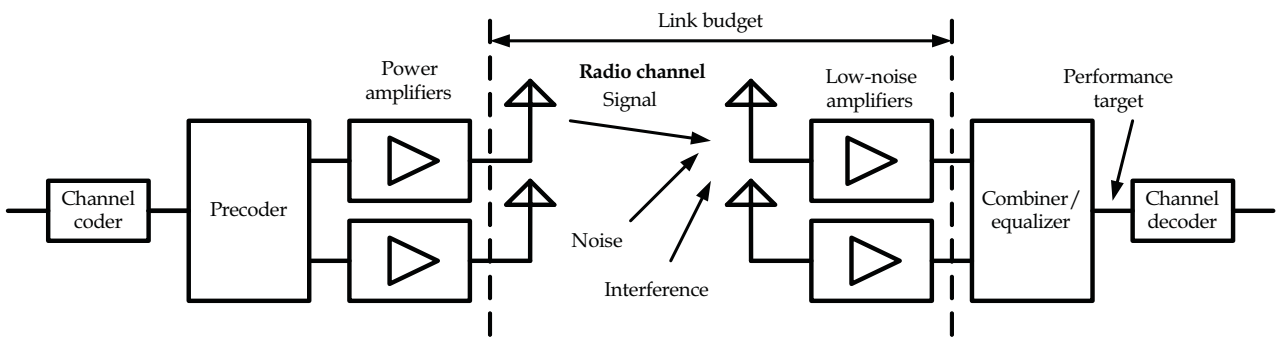

Fig. 1. Definition of a link budget

It is well known that energy is conserved in all physical processes. In communications, we are not especially interested in transmitting energy, but without energy, communication is not possible, and the performance of the receiver depends in part on the ratio of the received energy per bit to the noise power spectral density. Furthermore, it is not possible to observe the state of the channel without transmitting energy.

The physical channel is normally assumed to be linear (Bello, 1963; Proakis, 2001), but it may distort the transmitted signal because of temporal, frequency, and spatial selectivity. The noise is assumed to be additive white and Gaussian (AWGN). Some interfering signals from other users may also be received. Usually the transmitted signal is divided into blocks called frames, each containing multiple symbols, and each symbol includes one or more bits. The modulation and coding methods are taken into account in the received SNR required to attain the specified bit error rate or block error rate. Under worst case conditions, the raw bit error rate before channel decoding should be between $10^{-2}$ and $10^{-3}$. Then, with a good channel coding scheme, it is possible to reduce the bit error rate to less than say $10^{-6}$.

In some power-controlled systems the transmitted energy is zero when the channel is too weak. We must divide the systems into power-limited and energy-limited. Power-limited systems include, for example, base stations that are connected to the power-distribution network. Energy-limited systems include mobile terminals based on batteries. In such systems a fundamental question is how many bits can we reliably transmit for the expenditure of a given transmitted energy, which normally forms a major part of the total energy used in the transmitter. Thus the average transmitted energy per bit is a critical parameter.

In power-limited systems the power is averaged over all time, but in energy-limited systems the energy is averaged only when the transmitted energy is nonzero (Mämmelä et al., 2010). We refer to the latter kind of averaging as averaging with expurgation. Because of discontinuous transmission in a fading channel, the number of received symbols is a random variable and analysis becomes more difficult for energy-limited systems than in the power-limited case. If transmission is continuous, expurgation has no effect and there is no difference between power-limited and energy-limited systems.

Normally the received symbol energy is optimized to yield sufficiently good performance. In adaptive systems the changes in the channel must be slow compared to the symbol rate (Monsen, 1980; Proakis, 2001), which is valid if the coherence time of the channel is much 
larger than the symbol interval. This is a reasonable assumption when the bit rate is high and the symbol interval is short. In practice, because of the use of channel estimators whose observation interval is several symbol intervals, the channel should be essentially invariant during each block of symbols.

In this chapter we briefly review the major loss factors included in link budgets. We want to provide an intuitive explanation for these factors and introduce a new one. More specifically, we introduce a factor arising from the correlation between two loss factors. Such a situation is common in power-controlled systems where the transmitted energy and the channel energy gain $G=E_{\mathrm{r}} / E_{\mathrm{t}}$ are correlated where $E_{\mathrm{r}}$ is the received energy and $E_{\mathrm{t}}$ is the transmitted energy. If $E_{\mathrm{t}}=0$, we define $G=0$. The correlation is usually ignored in link budgets although it has a significant effect, either positive or negative, depending on the power control rule. The analytical background for this is explained in (Mämmelä et al., 2010). There can be also some other nonlinear interactions between the gains.

This chapter is organized as follows. We first summarize the required decision-theoretic tools to properly formulate link budgets. Next we discuss path loss, shadowing, and multipath fading of the channel and the corresponding fade margins. Other loss factors are briefly mentioned. Some channels are reciprocal and the requirements for this are discussed. Next we discuss the effect of correlation between different loss factors. Various averaging methods are used in the literature, but not all of them are appropriate for our purposes. Normalization of the channel model may be problematic since some models have an infinite peak energy gain, which obviously is inconsistent with the energy conservation law and may sometimes lead to confusing results as explained in (Xiang \& Pietrobon, 2003).

\section{Decision-theoretic tools}

Alternative approaches for formulating link budgets are based on rational decision-making under strict uncertainty (French, 1986, pp. 33-60). The first approach is called the max-min approach approach, which is sometimes called Wald's rule (French, 1986, p. 36). We use this approach when the distributions of random variables are unknown and we maximize the worst case performance. This approach is used in conventional link budgets, which are based in various margins such as fade and interference margin. Another approach called Laplace's principle of insufficient reason (French, 1986, p. 38) can also be used. Laplace argues in his "Essai philosophique des probabilités" that knowing nothing at all about the true state of nature is equivalent to all states having equal probability (French, 1986, p. 38). Following Laplace's line of reasoning leads to the assumption that all random variables whose probability distributions are unknown to the system designer are uniformly distributed random variables. Under the uniform distribution assumption, maximization of average performance is well justified (French, 1986, p. 49). The third approach is called the safety first approach. There are three schemes in this approach. The schemes are summarized in (Kotelba \& Mämmelä, 2008) where the original references can be found. The first scheme was devised by Roy. Here we minimize the probability of outage $\alpha$ for a given threshold $c$ set for the received SNR $\gamma_{\mathrm{r}}$, i.e., $\alpha=\min \operatorname{Pr}\left(\gamma_{\mathrm{r}}<c\right)$. This leads to the search for a probability density function $p\left(\gamma_{\mathrm{r}}\right)$ such that the ratio $\left(\mu_{\gamma}-c\right) / \sigma_{\gamma}$ is maximized where $\mu_{\gamma}$ is the mean of $\gamma_{\mathrm{r}}$ and $\sigma_{\gamma}$ is its standard deviation (Roy 1952, p. 434). The second scheme was derived by Kataoka. Here we set the probability of outage at a certain level and maximize the threshold for the received SNR, thus $\max _{c} \operatorname{Pr}\left(\gamma_{\mathrm{r}}<c\right)=\alpha$. The third scheme was published by Telser. In this scheme we maximize the mean of the received SNR for a given outage probability, i.e., 
$\max \left\{E\left\{\gamma_{\mathrm{r}}\right\}, \operatorname{Pr}\left(\gamma_{\mathrm{r}}<c\right)=\alpha\right\}$. The approaches lead to the conclusion that as an alternative to the worst case analysis, we can also propose average analysis based on an outage probability.

\section{Path loss of the channel}

According to the energy conservation law, the energy gain of a channel is always equal to or less than unity. The loss is the inverse of the gain, and it is usually expressed in decibels. To simplify our discussion we often use the term gain although in general the gain is smaller than unity. The gain has temporal, frequency, and spatial selectivity. Therefore the average gain depends on the distribution of the transmitted signal in these domains (Mämmelä et al., 2010). The gain is not only a property of the channel, but depends on how well the transmitted signal is "matched" to the channel, which may be randomly changing. In general, the gain is larger if the transmitted signal is using those parts of the channel in time, frequency, and space where the gain is the largest.

The path loss is the average loss at a given distance. It consists of the free-space loss, the absorption and diffraction loss because of the intervening medium, and the loss because of the reception on many multipath components due to reflections and refractions (Rappaport, 2002). In some specific scenarios the path loss can be smaller than the free-space loss because of the additional energy received from multipath components as in a waveguide, corridor, or duct.

\subsection{Free-space loss}

The concept of free-space loss is based on a model of an isotropic antenna in a vacuum. The free-space gain of an isotropic antenna is $G_{\mathrm{fsi}}=P_{\mathrm{r}} / P_{\mathrm{t}}=A_{\mathrm{i}} / A_{\mathrm{ro}}=(\lambda / 4 \pi r)^{2}$ (Saunders, 1999, $\mathrm{p}$. 68). It depends on the wavelength $\lambda$ and thus on the frequency $f=c / \lambda$. Here $A_{\mathrm{i}}=\lambda^{2} /(4 \pi)$ is the effective aperture of the isotropic antenna, and $A_{\mathrm{ro}}=4 \pi r^{2}$ is the area of the surrounding sphere of radius $r$. Intuitively there should be no dependence on wavelength. The energy is distributed in free space in the same way in all directions at all frequencies. The size of the antenna is assumed to be very small compared to the wavelength so that the antenna has an isotropic antenna pattern. The effective aperture of an isotropic antenna depends on the wavelength. Thus the smaller the wavelength and the higher the frequency, the smaller the effective aperture. We can imagine a situation where the transmitter antenna in a vacuum is surrounded by $\left\lfloor 1 / G_{\mathrm{fsi}}\right\rfloor$ receiver antennas in such a way that almost all the transmitted energy is collected. In that case the loss does not depend on the wavelength in free space, and in fact there is no free-space loss.

Physical antennas have an effective aperture $A_{\mathrm{e}}=G_{\mathrm{a}} \cdot \lambda^{2} /(4 \pi)$ where $G_{\mathrm{a}}$ is the gain of the antenna. The gain of a practical antenna depends on direction because of its finite size, and we usually refer to the highest gain. The gain is also frequency-dependent. The free-space gain between two antennas is specified as

$$
G_{\mathrm{fs}}=G_{\mathrm{t}} G_{\mathrm{r}}(\lambda / 4 \pi r)^{2}
$$

where $G_{t}$ and $G_{r}$ are the gains of the transmitter and receiver antennas. This is called Friis's equation (Saunders, 1999, pp. 69, 93-94). We have assumed that the gain is in the direction of the receiver at the transmitter and in the direction of the transmitter at the receiver, as otherwise there is an additional loss. Free-space loss can be approximated in practice when several Fresnel's zones defined by the transmitter and receiver are free around the antennas (Saunders, 1999, p. 49-50). 


\subsection{Absorption and diffraction loss}

In any medium, part of the transmitted energy is absorbed and diffracted, for example due to different material objects (Saunders, 1999, pp. 45-55, 127-135). In addition, at certain frequencies the radio waves are highly attenuated because of gas molecules and rain. Energy is often changed to heat in absorption. Energy is absorbed because the electrical particles, for example electrons, start to move in the medium, and the energy is partially changed to heat that cannot be received by an antenna but is distributed throughout the environment. In general the absorption loss of radio waves is increased when the wavelength is made shorter. Material objects cause also another phenomenon called diffraction. Thus part of the radio waves may be bent near the objects. Diffraction is stronger at lower frequencies. Absorption and diffraction losses are difficult to predict analytically especially if the form of the objects is irregular. Such losses are usually estimated by measurements (Pahlavan \& Levesque, 1995; Rappaport, 2002).

\section{Shadowing and multipath fading}

Fading is caused by shadowing and multipath propagation. The time-variant part of the absorption and diffraction loss is called shadowing. In general it is a much slower phenomenon than multipath fading. When the receiver is moved at a given distance from the transmitter, the medium seen by the radio waves is changed. In urban areas the coherence distance is in the order of ten meters (Gudmunson, 1991), which corresponds to tens or hundreds of wavelengths. In multipath fading the coherence distance may be less than a wavelength.

Under the worst case analysis, fading is normally included in link budgets as a margin. We are interested in the received SNR in the worst case in a slowly fading channel. As will be seen, typical fading models are such that the gain can approach zero. In practice we must accept a certain confidence level, say 90-99\%, with which the required received SNR is attained. If the SNR becomes lower, such a situation is called an outage. The corresponding outage probabilities are between $10 \%$ and $1 \%$. In mobile communications, typical edge-ofcell margins are such that the confidence level is $90 \%$ (Saunders, 1999, p. 183).

\subsection{Shadowing}

Shadowing is normally assumed to be frequency-nonselective, but in practice it is frequency-selective (Coulson et al., 1998). In a lossy medium the frequency has some effect because usually it is the thickness of the material in terms of the number of wavelengths and the electrical properties of the material that cause the loss (Pahlavan \& Levesque, 1995). We need a database where the materials are classified according to their electrical properties and the losses are normalized to wavelength. The loss caused for example by a wall can then be estimated by measuring its thickness in wavelengths. Not all the multipath components propagate directly to the receiver. Different multipath components see a different absorption and diffraction loss, and therefore shadowing is frequency-selective.

Shadowing can be divided into global and local shadowing (Salo, 2006, pp. 33-35). Local shadowing refers to changes in the absorption and diffraction loss when the receiver moves in a given environment without changing its distance to the transmitter. A model for local shadowing should be used in link simulations. Based on the central limit theorem we usually assume that local shadowing is lognormal. The logarithm of the product of several independent gain factors $G_{k}=\log \left[\Pi_{i}\left(G_{i}\right)\right]=\Sigma_{i} \log \left(G_{i}\right)$ is normally distributed if the 
assumptions of the theorem are valid. The measured standard deviations are usually in the order of 3-6 dB.

Global shadowing refers to the changes in the absorption loss globally at a given distance. Those changes are much larger than in local shadowing since there can be large differences in the media. In a link budget global shadowing is pessimistic and is based on the assumption that we do not know much about the intervening medium. If we know the materials comprising the medium and their location, we can improve the estimate. Global shadowing is often assumed to be lognormal, but strictly speaking it cannot be lognormal if local shadowing is lognormal. The average of $K$ lognormal random variables $G=(1 / K) \Sigma_{k} G_{k}$ is not in general lognormal, and in fact, according to the central limit theorem it tends to be normal when $K$ is large. However, there is a more or less general consensus that the average of independent lognormal random variables can be approximated by another lognormal random variable with appropriately chosen parameters (Stüber, 2001, pp. 129-139). The measured standard deviations are in the order of 5-12 dB (Stüber, 2001, p. 21).

\subsection{Multipath fading}

Multipath propagation is caused by reflection and scattering of radio waves in flat and rough zones between two media (Sounders, 1999). Multipath propagation can also be caused by deterministic or random changes in the refractive index of the medium such as the atmosphere. The fading caused by multipath propagation is best explained by using a sinusoidal signal. A physical distance $d$ causes a delay $\tau=d / c$ and shifts the phase by $\varphi=$ $-2 \pi f \tau$. Multipath signals can have path-length differences of several kilometres (Turin, 1980; Pahlavan \& Levesque, 1995). For example, a path-length difference of $6 \mathrm{~km}$ corresponds to a delay difference of $20 \mu$ s. Thus usually the path length difference $\Delta d$ is in the order of several wavelengths, and the magnitude of the phase shift difference is $|\Delta \varphi|>>2 \pi \mathrm{rad}$. Therefore, the signals are added together in the receiver antenna either constructively when the phase difference $|\Delta \varphi| \approx 2 n \pi$ where $n$ is an integer or destructively when $|\Delta \varphi| \approx(2 n+1) \pi$, depending on the phase relationship. The phase shift $\varphi$ is usually frequency-dependent, and thus multipath fading is frequency-selective. If $|\Delta \varphi|<<2 \pi \mathrm{rad}$, the multipath fading would disappear, but this is not normally the case because usually $\lambda \ll|\Delta d|$. The fading is also time-selective since the delays are constantly changing due to terminal mobility.

The amplitude distribution of multipath fading is usually assumed to be Rayleigh or Rician (Proakis, 2001). These distributions arise due to the central limit theorem. The received power has then either a central or noncentral chi-square distribution. Sometimes also the Nagakami- $m$ distribution also used for the amplitude distribution. The latter corresponds to a generalized Rayleigh distribution (Proakis, 2001, p. 45-48).

Energy does not disappear in multipath fading. The energy is spatially distributed and in different locations the receiver antenna sees a different amount of energy. The channel is thus also spatially selective. In theory all that energy could be collected if there were no absorption.

\subsection{Beam forming and antenna diversity}

Often transmitters and receivers use more than one antenna element. Such systems may be classified as beam forming or diversity systems. Beam forming can be seen to be more general than antenna diversity. In beam forming significant aliasing in the spatial or directional domain is avoided by using the sampling theorem in space. The maximum distance between antenna elements is half a wavelength. The antenna pattern can be 
modified by complex weighting. The finite size of the antenna array causes side lobes similar to the Gibbs phenomenon in filters (Hansen, 1966). Thus there is some aliasing because of the side lobes.

In beam forming we process correlated signals to or from certain directions. In receiver beam forming we make the effective aperture of the receiver larger and thus we receive more energy. In transmitter beam forming the spreading loss is made smaller because the same energy is focused in a given direction. If the number of antenna elements is $N$, the maximum antenna array gain is $10 \log _{10} N$ (Parker \& Zimmermann, 2002). For example, if $N$ $=16$, the gain is $12 \mathrm{~dB}$. Directional antennas can be used to reduce multipath components.

In multiple-input multiple-output (MIMO) diversity systems the distance between antenna elements is so large that we ideally process uncorrelated signals coming from all directions. This is called rich scattering. The distance is in the order of wavelengths. Antenna diversity is a special case of beam forming in the sense that some of the complex weights and the antenna elements are removed and aliasing is introduced. In the transmitter the signals are made orthogonal by using special coding. In the receiver the signals may be combined coherently as in maximal ratio combining. In antenna diversity the antenna array has an antenna pattern that looks random and it is constantly changing when the multipath propagation is changing. Because of aliasing we cannot freely modify the antenna pattern. One direction corresponds to many directions and the antenna pattern has some periodicity in different directions.

If we add the number of transmitter antennas in antenna diversity, the received energy is not in general increased since it must be divided among the antennas and the transmitted energy is the basic resource (Schwartz et al., 1966, pp. 555-558). The antenna pattern is approximately omnidirectional although random, and there is no array gain on average. In diversity systems, there is typically no array gain, but there is usually element gain. In receiver diversity the situation is different because the effective aperture is increased by using two or more antennas.

\section{Other loss factors and margins and reciprocity}

Antenna efficiency is defined as the total radiated energy divided by the total input energy. The efficiency depends on frequency. Normally the antenna radiates with the highest efficiency at frequencies where the wavelength is of the same order as the physical dimensions of the antenna. A physical antenna thus acts as a band-pass filter.

Additional loss factors include for example base station feeder loss, body and matching loss, and implementation loss (Saunders, 1999; Sklar, 2001). Some loss factors are not easy to estimate and thus it is common to use specific margins, for example interference margin (Saunders, 1999). Interference margin is especially important for cell edge performance in cellular systems.

Reciprocity is usually assumed in time-division duplex systems, particularly when the channel is changing sufficiently slowly. Antenna elements are in general reciprocal between the transmitter and receiver (Saunders, 1999, pp. 66-68). This means that the impulse response of the channel does not depend on direction. If we use a set of antenna elements, the situation may be different since we may weight the signals in different ways in the transmitter and receiver. Also the noise and interference depend on the environment and they are not in general reciprocal. Thus the SNR is not in general reciprocal. Electronic components such as power amplifiers are usually not reciprocal. 


\section{Correlation between gain factors}

The gain factors are assumed to be multiplicative. Thus the logarithm of the total gain of the form $G=\Pi_{i}\left(G_{i}\right)$ is $\log (G)=\log \left[\Pi_{i}\left(G_{i}\right)\right]=\Sigma_{i} \log \left(G_{i}\right)$ where the gains $G_{i}$ are deterministic. If the gains are correlated random variables, this approach is no longer valid. For brevity, we consider two gains $G_{1}$ and $G_{2}$, which are correlated with the $\operatorname{covariance} \operatorname{Cov}\left\{G_{1} G_{2}\right\}=E\left\{\left[G_{1}-\right.\right.$ $\left.\left.E\left\{G_{1}\right\}\right]\left[G_{2}-E\left\{G_{2}\right\}\right]\right\}=E\left\{G_{1} G_{1}\right\}-E\left\{G_{1}\right\} E\left\{G_{2}\right\}$, where $E$ refers to the expected value. The total instantaneous gain is $G=G_{1} G_{2}$, but the average gain is

$$
E\{G\}=E\left\{G_{1} G_{2}\right\}=E\left\{G_{1}\right\} E\left\{G_{2}\right\}+\operatorname{Cov}\left\{G_{1}, G_{2}\right\} .
$$

The covariance in (2) can be positive, negative, or zero.

A good example is the covariance between the transmitted energy and the channel energy gain in a power-controlled transmitter. We have shown in (Mämmelä et al., 2010) that the covariance has a significant effect on the link budgets. The basic power control rules are water-filling, which maximizes capacity if the bit rate is optimally adjusted at the same time, and truncated channel inversion, which minimizes the outage probability for a given transmitter power constraint and bit error rate (Biglieri et al., 1998, pp. 2627-2628). Under water-filling we transmit more energy when the channel is good, but when the channel is sufficiently bad, we do not transmit at all. In this way the transmitted signal is well matched to the channel, and we actually receive a good fraction of the transmitted energy. The method is optimum only for isolated links due to possible interference to other users. In truncated channel inversion we do the opposite: we transmit more energy when the channel is bad, but if the channel is too bad, we do not transmit at all. In full channel inversion we transmit all the time. These alternatives can be approximated by recursive algorithms (Gilhousen et al., 1991; Caire et al., 1999; Yu et al., 2004; Höyhtyä \& Mämmelä, 2007).

In practice it is easier to track shadowing which is much slower than multipath fading. However, this depends on the system parameters. There is no fundamental reason why we cannot track multipath fading if it is slow enough compared to the symbol rate of the system.

The covariance can be included in the link budgets as an additional gain or loss because the average received energy can be expressed as

$$
E\left\{E_{\mathrm{r}}\right\}=E\left\{E_{\mathrm{t}} G\right\}=E\left\{E_{\mathrm{t}}\right\} E\{G\}+\operatorname{Cov}\left\{E_{\mathrm{t}}, G\right\} .
$$

In practice, a more informative figure of merit is the efficiency of power transmission defined as

$$
\eta=E\left\{E_{\mathrm{r}}\right\} /\left(E\left\{E_{\mathrm{t}}\right\} E\{G\}\right)=1+\operatorname{Cov}\left\{E_{\mathrm{t}}, G\right\} /\left(E\left\{E_{\mathrm{t}}\right\} E\{G\}\right) .
$$

Here is a short interpretation of (3) and (4). The covariance $\operatorname{Cov}\left\{E_{\mathrm{t}}, G\right\}$ captures the effect of adaptive power control on the average received signal energy, and therefore, on the link energy budget. The efficiency parameter $\eta$ of (4) describes whether there is an additional gain or loss compared to the traditionally used approach where possible correlation between the transmitted energy and channel gain is not taken into account.

We illustrate the effect of the covariance at two extremes. The covariance can be either maximized or minimized with respect to some additional constraints on the moments of distribution of transmitted energy (Kotelba \& Mämmelä, 2010b). For given average transmitted energy $E\left\{E_{t}\right\}$ and representative energy gain $E\{G\}$, the maximization of the 
covariance value is equivalent to maximization of the average received energy or maximization of transmission efficiency. The representative energy gain is the average energy gain of a signal whose energy is uniformly distributed in all the domains (Mämmelä et al., 2010). Similarly, minimization of the covariance value is equivalent to minimization of the average received energy or minimization of transmission efficiency. We demonstrated in (Kotelba \& Mämmelä, 2010b) that to maximize the average received energy $E\left\{E_{r}\right\}$ the instantaneous transmitted energy $E_{\mathrm{t}}$ should be proportional to the channel gain $G$. On the other hand, if the instantaneous transmitted energy $E_{\mathrm{t}}$ is inversely proportional to the square of the channel gain $G$, then the average received energy $E\left\{E_{\mathrm{r}}\right\}$ is minimized. In general there is some gain for water-filling and some loss for truncated channel inversion, compared to the case where the transmitted energy is constant (Mämmelä et al., 2010). The difference can be several decibels depending on the transmitted signal-to-noise ratio. A good way to include the effect of the covariance in the average link budget is to use the transmitted SNR instead of the received SNR in bit error rate measurements. The transmitted SNR $\gamma_{t}$ is defined to be the ratio of the transmitted energy per bit and the received noise spectral density (Mämmelä et al., 2010).



Fig. 2. Covariance between instantaneous transmitted energy and channel gain for various adaptive power control schemes

In the numerical examples in Figs. 3 through 5 we did not use expurgation, which implies that the results are valid for power-limited systems. In nonexpurgated systems the analysis is tractable and we are able to illustrate the concepts. In our numerical results, shown in Figs. 2 and 3, we used a composite gamma/lognormal fading channel model (Simon \& Alouini, 2000, pp. 24-25) with multipath fading parameter $m=4$, shadowing channel gain $\mu$ 
$=0 \mathrm{~dB}$, and shadowing standard deviation $\sigma=4 \mathrm{~dB}$. For truncated channel inversion, the cut-off level was set to zero, that is, we considered full channel inversion.

The results shown in Fig. 3 can be used in average link budgets. In the worst case analysis we must define an outage probability and determine how far below the mean the SNR can be. The cumulative distribution function (cdf) $F\left(\gamma_{\mathrm{r}}\right)$ of the received SNR $\gamma_{\mathrm{r}}$ is shown in Fig. 4. Three power control rules are used in a composite multipath/shadowing channel. The average transmitted SNR is constant for all power control rules and equal to $5 \mathrm{~dB}$. The cutoff value in truncated channel inversion $\mu_{\mathrm{tci}}$ was set to 0.223489 to meet the outage probability requirement. The respective averages are marked with an asterisk. Water-filling gives the largest average received SNR (positive covariance), followed by no power control (zero covariance), and truncated channel inversion (negative covariance). For a target outage probability of $10 \%$, the truncated channel inversion and water-filling require the smallest and the largest fade margin, respectively.

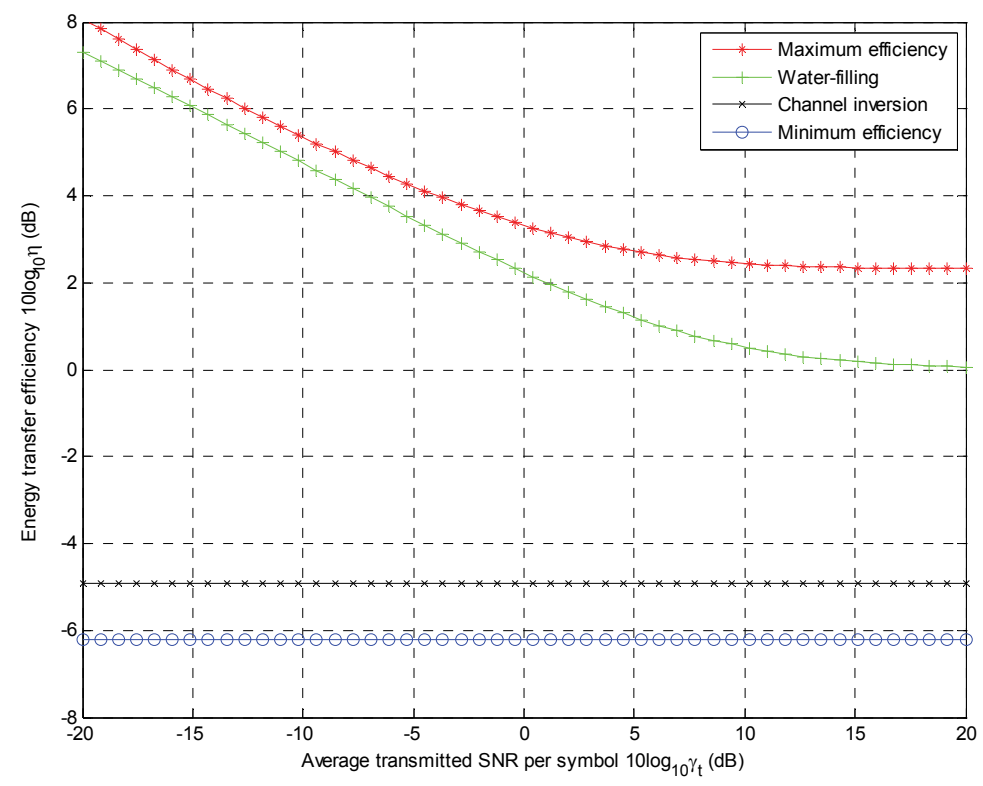

Fig. 3. Efficiency of power transmission in a composite multipath/shadowing channel

The corresponding fade margins are shown in Fig. 5, again for a target outage probability of $10 \%$. The results in Fig. 5 suggest that under the water-filling power control rule, the maximum outage probability requirement of $10 \%$ can be satisfied only for sufficiently large average transmitted SNR. Below a certain value of the average transmitted SNR, the outage probability associated with a nonzero cutoff value $\mu_{\mathrm{wf}}$ exceeds the target probability of $10 \%$. Since the water-filling cutoff value $\mu_{\mathrm{wf}}$ is unique for a given average transmitted SNR, it is not possible to meet the maximum outage probability requirement and the required fade margin tends to infinity. This effect is illustrated in Fig. 5. The results in Fig. 5 can be used in the worst case analysis when we add the efficiency of power transmission shown in Fig. 3 
and the fade margin shown in Fig. 5. In conventional link budgets the fade margin for no power control is used. Thus we have provided some tools to include power control in both the average and worst case analyses.

\section{Averaging}

In averaging we must consider possible commutability problems. We must know in which domain we must perform averaging. The expectation operation commutes over linear and affine transformations of the form $\mathbf{y}=\mathbf{A x}+\mathbf{c}$, i.e., $\mathrm{E}\{\mathbf{y}\}=\mathbf{A} \mathrm{E}\{\mathbf{x}\}+\mathbf{c}$ where $\mathbf{x}$ is a random column vector, $\mathbf{A}$ is a constant matrix, and $\mathbf{c}$ is a constant column vector (Kay, 1993, pp. 149$150,349-350,390$ ). If $\mathbf{c}=\mathbf{0}$, the transformation is linear, and the superposition theorem is valid, and if $\mathbf{c} \neq \mathbf{0}$, the transformation is affine. The property for the expectation operation does not in general carry over to nonlinear transformations, for example to those including logarithms and inversions. The problem is discussed in (Kay, 1993, pp. 173-177, 185) for common estimators.

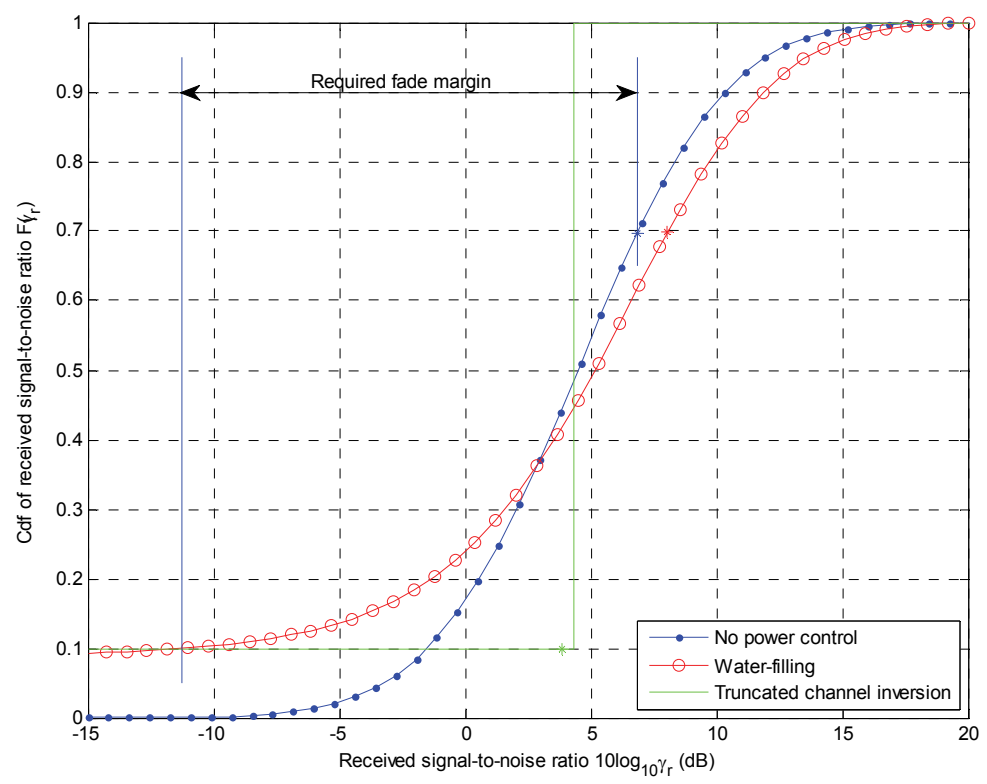

Fig. 4. Cdf's of the received SNR for no power control, water-filling, and truncated channel inversion

When we measure the attenuation of the channel, the received power or energy must be averaged and compared with the transmitted power or energy. By definition, averaging should be done in the linear domain, i.e., in watts or joules. However, sometimes it is more practical to average in the logarithmic domain using decibels since most link budgets are computed in decibels. The two averages are not in general identical even if expressed in the same domain (Pahlavan \& Levesque, 1995), and thus averaging of decibels may be 
misleading. By using Jensen's inequality (Feller, 1971, p. 153) we have $E\{\log X\} \leq \log E\{X\}$. In general logarithmic averaging leads to pessimistic results and the estimated average gain is lower than the actual average gain.

In the literature the local shadowing is usually averaged in the linear domain and the global shadowing is averaged in the logarithmic domain (Rappaport 2002, pp. 139-141). If we assume that the lognormal approximation is good enough, the global average is actually the median in the linear domain, not the true linear average. If we do not use the average itself but the confidence limits in the worst case analysis, there is no problem with this approach. In a fading channel we must often average ratios of the form $X / Y$ where $X$ and $Y$ are random variables and $Y \neq 0$. Now $E\{X / Y\}=E\{X \cdot 1 / Y\}=E\{X\} E\{1 / Y\}+\operatorname{Cov}\{X, 1 / Y\}$. If $X$ and $1 / Y$ were uncorrelated, we would have $E\{X / Y\}=E\{X\} \cdot E\{1 / Y\}$. In general $E\{X / Y\} \neq$ $E\{X\} / E\{Y\}$ where $E\{Y\} \neq 0$. Such ratios must be considered for example when the received energy $X=E_{\mathrm{r}}$ and transmitted energy $Y=E_{\mathrm{t}}$ are random in a power-controlled system, and we must estimate the average energy gain of the channel. The variables $X$ and $1 / Y$ are also correlated in this case. Another example is when the transmitted energy per block is $X=E_{\mathrm{t}}$ and the number of bits in a block $Y=w$ is a random variable and we must estimate the average transmitted energy per bit $E\left\{E_{\mathrm{t}} / w\right\}$ (Mämmelä et al., 2010). In such cases we obtain different results with different averaging methods $E\{X / Y\}$ or $E\{X\} / E\{Y\}$ and we must decide what we really want. In general, the observation interval should be sufficiently large that the random variable $Y$ in the denominator can be assumed to be essentially constant. In such cases $E\{X / Y\} \approx E\{X\} / E\{Y\}$.

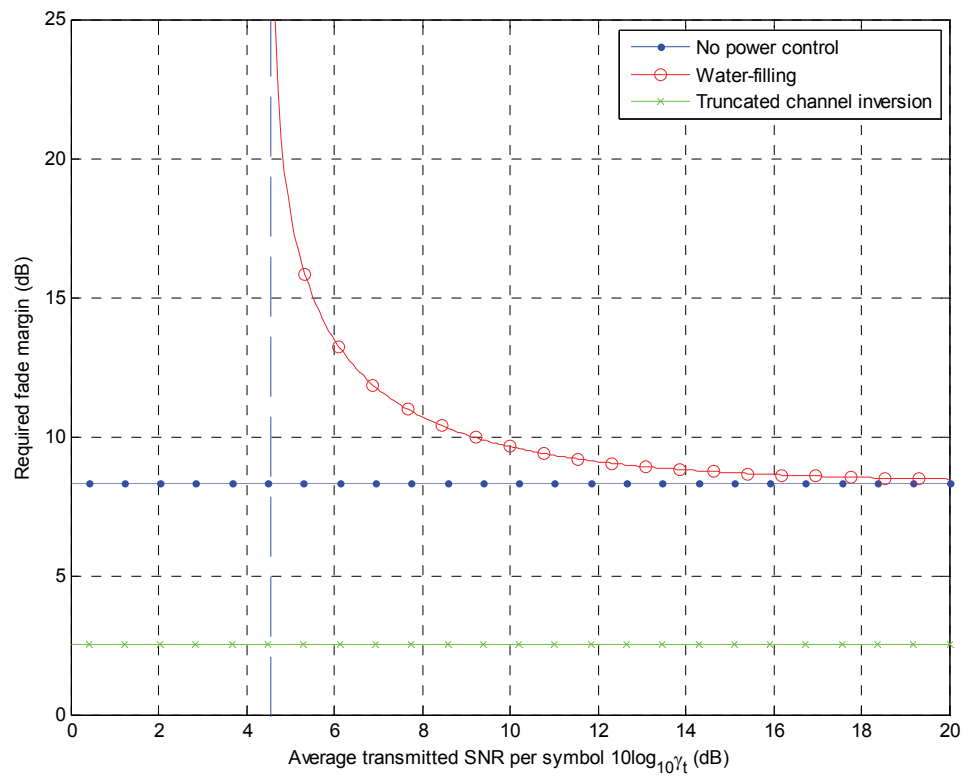

Fig. 5. Fade margins for no power control, water-filling, and truncated channel inversion 
In a fading channel transmission may not be continuous. In such cases we must distinguish between power-limited and energy-limited systems. In energy-limited systems expurgation is used to measure the average transmitted energy and the corresponding SNR. In powerlimited systems no expurgation is used for the SNR. In all systems expurgation is used when measuring the bit error rate since we are not interested in bits that are not actually transmitted. When using expurgation, we must know when the actual bits are received.

\section{Normalization of the channel}

The average energy gain of the channel depends on the signal structure: how the energy is distributed in the time, frequency and spatial domains and thus how well the transmitted signal is matched to the channel (Mämmelä et al., 2010). In link budgets the channel is usually normalized according to the so-called representative energy gain. For example, if the channel model has the form $Y=a X$, the representative energy gain is $E\left\{|a|^{2}\right\}$. Some channel models are such that their instantaneous energy gain is infinite although the average energy gain would be finite. Normally this does not matter since the path loss is rather large, and it is quite improbable that the instantaneous gain would be larger than the path loss. Strictly speaking our models should be truncated. If our aim is to compare the effect of different distortions, it may be reasonable to normalize the models according to the peak energy gain (Xiang \& Pietrobon, 2003).

If in an antenna diversity system we add new receiver antennas at the same distance from the transmitter, the received energy would be increased linearly. In principle we can increase the effective aperture up to the whole sphere surrounding the transmitter antenna. We could have a suitably formed effective aperture that fits together like a jigsaw puzzle. We receive only part of the transmitted energy since part of the energy is absorbed and transformed to other types of energy. The received energy would saturate to that maximum and no longer increases linearly with the number of antenna elements.

The measured path loss includes all the multipath signal components, not just the direct path component. If directive antennas were to be used, some multipath components would be rejected. By including the multipath components we are able to clarify the normalization problem we have. In fair comparisons in energy-limited systems the starting point is the basic resource or the average transmitted energy per bit. If we have different channel models, we have the problem of how we should normalize them. Intuitively peak normalization would be fair. However, the theoretical peak is not always attained. For example, in a multipath channel the multipath components experience certain delays. In theory the peak would be such that all the multipath components are added constructively. This is not always possible if we cannot select the delays arbitrarily. A certain delay corresponds to a certain phase shift. For example, there can be additional phase shifts due to reflections. In general, the peak of the square of the magnitude of the transfer function of a slowly fading channel is less than or equal to the theoretical maximum.

Many channel models cannot be peak-normalized since the peak energy gain is unlimited. Such models include for example Rayleigh, Rician and lognormal fading channels. In such channels surprisingly there appears a St. Petersburg-like paradox (Kotelba \& Mämmelä, 2010a). It can be resolved by using suitable performance metrics and fading models. Normalization is further discussed in (Loyka \& Levin, 2009). 


\section{Conclusion}

Link power or energy budgets are usually used to estimate the received SNR and thus the expected performance of the receiver. In a link budget we evaluate the different loss factors in a communication system. Usually the link budget is computed in the logarithmic domain and the different factors are added together in decibels. Normally a worst case analysis is made. We have described the factors in detail and have shown the uncertainties we have, and how to possibly reduce those uncertainties. We have also provided tools describing how to include power control in the worst case analysis or in the alternative average analysis.

A general assumption is that there are no nonlinear interactions between the different factors. When power control is used there is a strong correlation between the energy gain of the channel and the transmitted energy. Numerical results were presented to show the effect of this correlation. The chapter has concentrated mainly on single isolated links. An extension to multiple links would be useful, but so far no standard practices are available other than the interference margins.

We have also shown the limitations of the common fading channel models leading to some pathological phenomena that never occur in practical channels. We discussed the normalization of the channel models and averaging of the power and energy in powerlimited and energy-limited systems. The emphasis was in phenomena that are not very well known in the scientific society.

\section{References}

Bello P. A. (1963). Characterization of randomly time-variant linear channels, IEEE Transactions on Communications Systems, Vol. CS-11, No. 4, pp. 360 - 393.

Biglieri E., Proakis J., and Shamai S. (1998). Fading channels: Information-theoretic and communications aspects, IEEE Transactions on Information Theory, Vol. 44, No. 6, pp. 2619 - 2692.

Caire G., Taricco G., and Biglieri E. (1999). Optimum power control over fading channels, IEEE Transactions on Information Theory, Vol. 45, No. 5, pp. 1468-1489.

Chu T.-S. and Greenstein L. J. (1999). A quantification of link budget differences between the cellular and PCS bands, IEEE Transactions on Vehicular Technology, Vol. 48, No. 1, pp. $60-65$.

Coulson A. J., Williamson A. G., and Vaughan R. G. (1998). A statistical basis for lognormal shadowing effects in multipath fading channels, IEEE Transactions on Communications, Vol. 46, No. 4, pp. 494-502.

Feller W. (1971). An Introduction to Probability Theory and Its Applications, Vol. 2, 2nd ed., John Wiley \& Sons, New York.

French S. (1986). Decision Theory: An Introduction to the Mathematics of Rationality. Halsted Press, New York.

Gilhousen K. S., Jacobs I. M., Padovani R., Viterbi A. J., Weaver L. A., Jr., and Wheatley C. E., III (1991). On the capacity of a cellular CDMA system, IEEE Transactions on Vehicular Technology, Vol. 40, No. 2, pp. 303-312. 
Gudmundson M. (1991). Correlation model for shadow fading in mobile radio systems, Electronics Letters, vol. 27, no. 23, pp. 2145 - 2146.

Hansen R. C. (1966). Microwave Scanning Antennas, John Wiley \& Sons, New York.

Höyhtyä M. and Mämmelä A. (2007). Adaptive inverse power control using an FxLMS algorithm, Proceedings of the IEEE Vehicular Technology Conference (VTC) Spring, Dublin, Ireland, pp. 3021-3025.

Kay, S. M. (1993). Fundamentals of Statistical Signal Processing: Estimation Theory. PrenticeHall, Englewood Cliffs, NJ.

Kotelba A. and Mämmelä A. (2008). Application of financial risk-reward theory to adaptive transmission, Proceedings of the IEEE Vehicular Technology Conference (VTC) Spring, Singapore, pp. 1756 - 1760.

Kotelba A. and Mämmelä A. (2010a). St. Petersburg paradoxes in performance analysis of adaptive wireless systems, Proceedings of the IEEE Vehicular Technology Conference (VTC) Spring, Taipei, Taiwan.

Kotelba A. and Mämmelä A. (2010b). Efficiency of power transmission in adaptive communication systems, unpublished.

Loyka S. and Levin G. (2009). On physically-based normalization of MIMO channel matrices, IEEE Transactions on Wireless Communications, Vol. 8, No. 3, pp. 1107-1112.

Lucky R. W., Saltz J., and Weldon E. J., Jr. (1968). Principles of Data Communication. McGrawHill, New York.

Mämmelä A., Kotelba A., Höyhtyä M., and Taylor D. (2010). Relationship of average transmitted and received energies in adaptive transmission, IEEE Transactions on Vehicular Technology, Vol. 59, No. 3, pp. 1257-1268.

Monsen P. (1980). Fading channel communications, IEEE Communications Magazine, Vol. 18, No. 1, pp. 16-25.

Pahlavan K. and Levesque A. H. (1995). Wireless Information Networks. John Wiley \& Sons, New York.

Parker D. and Zimmermann D. C. (2002). Phased arrays-Part I: Theory and architectures, IEEE Transactions on Microwave Theory and Techniques, Vol. 50, No. 3, pp. 678-687.

Proakis J. G. (2001). Digital Communications, 4rd ed., McGraw-Hill, New York.

Rappaport T. S. (2002). Wireless Communications: Principles and Practice, 2nd ed., PrenticeHall, Englewood Cliffs, NJ.

Roy A. D. (1952). Safety first and the holding of assets, Econometrica, Vol. 20, No. 3, pp. 431 449 .

Salo, J. (2006). Statistical analysis of the wireless propagation channel and its mutual information, Doctoral thesis, Helsinki University of Technology, Espoo, Finland.

Saunders S. R. (1999). Antennas and Propagation for Wireless Communication Systems. John Wiley \& Sons, West Sussex, UK.

Schwartz M., Bennett W. R., and Stein S. (1966). Communication Systems and Techniques. McGraw-Hill, New York.

Simon M. K. and Alouini M.-S. (2000). Digital Communication Over Fading Channels: A Unified Approach to Performance Analysis. John Wiley \& Sons, New York.

Sklar B. (2001). Digital Communications: Fundamentals and Applications, 2nd ed., Prentice-Hall, Upper Saddle River, NJ. 
Stüber G. L. (2001). Principles of Mobile Communication, 2nd ed., Kluwer Academic Publishers, Norwell, MA.

Turin G. L. (1980). Introduction to spread-spectrum antimultipath techniques and their application to urban digital radio, Proceedings of the IEEE, Vol. 68, No. 3, pp. 328 353.

Xiang W. and Pietrobon S. S. (2003). On the capacity and normalization of ISI channels, IEEE Transactions on Information Theory, Vol. 49, No. 9, pp. 2263-2268.

Yu W., Rhee W., Boyd S., and Cioffi J. M. (2004). Iterative water-filling for Gaussian vector multiple-access channels, IEEE Transactions on Information Theory, Vol. 50, No. 1, pp. 145-152. 




\author{
Vehicular Technologies: Increasing Connectivity \\ Edited by Dr Miguel Almeida
}

ISBN 978-953-307-223-4

Hard cover, 448 pages

Publisher InTech

Published online 11, April, 2011

Published in print edition April, 2011

This book provides an insight on both the challenges and the technological solutions of several approaches, which allow connecting vehicles between each other and with the network. It underlines the trends on networking capabilities and their issues, further focusing on the MAC and Physical layer challenges. Ranging from the advances on radio access technologies to intelligent mechanisms deployed to enhance cooperative communications, cognitive radio and multiple antenna systems have been given particular highlight.

\title{
How to reference
}

In order to correctly reference this scholarly work, feel free to copy and paste the following:

Aarne Mämmelä, Adrian Kotelba, Marko Höyhtyä and Desmond P. Taylor (2011). Link Budgets: How Much Energy is Really Received, Vehicular Technologies: Increasing Connectivity, Dr Miguel Almeida (Ed.), ISBN: 978-953-307-223-4, InTech, Available from: http://www.intechopen.com/books/vehicular-technologiesincreasing-connectivity/link-budgets-how-much-energy-is-really-received

\section{INTECH}

open science | open minds

\section{InTech Europe}

University Campus STeP Ri

Slavka Krautzeka 83/A

51000 Rijeka, Croatia

Phone: +385 (51) 770447

Fax: +385 (51) 686166

www.intechopen.com

\section{InTech China}

Unit 405, Office Block, Hotel Equatorial Shanghai

No.65, Yan An Road (West), Shanghai, 200040, China 中国上海市延安西路65号上海国际贵都大饭店办公楼 405 单元

Phone: +86-21-62489820

Fax: $+86-21-62489821$ 
(C) 2011 The Author(s). Licensee IntechOpen. This chapter is distributed under the terms of the Creative Commons Attribution-NonCommercialShareAlike-3.0 License, which permits use, distribution and reproduction for non-commercial purposes, provided the original is properly cited and derivative works building on this content are distributed under the same license. 